\title{
Laparoscopic Versus Open Enucleation for Solitary Insulinoma in the Body and Tail of the Pancreas
}

\author{
Constantine Karaliotas • George Sgourakis
}

Received: 4 January 2009 / Accepted: 12 June 2009 /Published online: 18 July 2009

(C) 2009 The Society for Surgery of the Alimentary Tract

\begin{abstract}
Background Insulinomas, benign in the vast majority, are the prevailing pancreatic endocrine tumors amenable to surgical resection which is beneficial in most instances. This study aimed to compare the results of laparoscopic vs. open surgery enucleation of insulinomas.

Methods From October 1999 to June 2008, 12 case series of enucleation for benign insulinoma in the body and tail of the pancreas were identified through retrospective review of medical records. Main outcome measures were recurrent hypoglycemia, conversion to open procedure, complications, and length of hospital stay.

Results Seven patients were addressed with open and five with laparoscopic procedure. Mean age was 55 years (36-69). Lesions were identified preoperatively (via computed tomography and endoscopic ultrasonography) in 5/7 in the open and $4 / 5$ in the laparoscopic group. Intraoperative ultrasound identified the rest of insulinomas. One conversion to the open approach was mandatory because the insulinoma was resting on the portal vein. The mean operative time and hospital stay was $92 \mathrm{~min}(66-126) / 14$ days (11-22) for the open and $121 \mathrm{~min}(89-187) / 11$ days (5-18) for the laparoscopic procedure (including conversion) $(p<0.5$ in both comparisons). Pancreatic fistula rate was respectively $28.57 \%(2 / 7)$ and $20 \%(1 / 5)$ $(p=0.65)$. Mortality was nil. Mean follow-up was 54 months (3-109). Recurrent hypoglycemia was documented in one patient of the laparoscopic group $(p=0.46)$ but blood glucose concentrations remained stable with diazoxide.

Conclusion Laparoscopic insulinoma enucleation seems to be a feasible and safe approach associated with reduction in hospital stay and comparable rates of pancreatic fistula in relation to open surgery.
\end{abstract}

Keywords Pancreatic insulinoma.

Laparoscopic enucleation · Intraoperative ultrasound

Electronic supplementary material The online version of this article (doi:10.1007/s11605-009-0954-z) contains supplementary material, which is available to authorized users.

C. Karaliotas $\cdot$ G. Sgourakis $(\bowtie)$

2nd Surgical Department and Surgical Oncology

Unit of "Korgialenio-Benakio", Red Cross Hospital,

11 Mantzarou str., Neo Psychiko,

15451 Athens, Greece

e-mail: ggsgourakis@yahoo.gr 